\title{
金/铜纳米异质界面的电荷转移等离激元调控
}

\author{
朱青青 宋晓君邩兆祥* \\ (中国科学技术大学化学系 中科院软物质化学重点实验室 合肥微尺度物质科学国家研究中心 合肥 230026)
}

\begin{abstract}
摘要 金属纳米结构由于其独特的局域表面等离激元共振现象而倍受关注, 对催化、传感、纳米医学以及光学器件等 具有重要意义. 电荷转移等离激元共振强烈依赖于纳米单元间的导电结点, 可产生频率连续可调的共振光吸收和光散 射, 为获得高度局域化的增强光磁场和光热效应提供了可能. 然而, 受制于已有构筑手段和有限的结构种类, 相关研 究仍处于初级阶段. 针对此, 本工作发展了一种十分简单、有效的 $\mathrm{Au} / \mathrm{Cu}$ 纳米异质结点调控策略, 利用廉价易得的天然 DNA 分子在金纳米粒子 “种子” 表面发生非特异性吸附，有效控制铜在金表面发生异相成核时的相间接触面积，得到 导电结点宽度连续可调的电荷转移纳米粒子二聚体. 实验光谱和理论模拟显示, 结点宽度、铜和金纳米粒子的尺寸是 决定电荷转移等离激元性质的重要参数, 其分别可由 DNA 吸附量、 $\mathrm{Cu}^{2+}$ 加入量和金纳米粒子尺寸加以控制, 进而实现 共振波长在可见至近红外区的宽广调节. 通过与其它吸附分子对比证明了 DNA 吸附调控模式的独特性. 这种具有可调 控导电结点的双金属纳米异质界面为实现电荷转移等离激元共振与催化和传感等功能的集成以及相关应用探索奠定 了重要基础.
\end{abstract}

关键词 电荷转移; 界面; 金属; 二聚体; DNA

\section{Tunable Charge Transfer Plasmon at Gold/Copper Heterointerface}

\author{
Zhu, Qingqing Song, Xiaojun Deng, Zhaoxiang* \\ (CAS Key Laboratory of Soft Matter Chemistry, Hefei National Research Center for Physical Sciences at the Microscale, \\ Department of Chemistry, University of Science and Technology of China, Hefei 230026, China)
}

\begin{abstract}
Metal nanostructures with localized surface plasmon resonance (LSPR) have attracted great attention in catalysis, sensing, nanooptics, and nanomedicine. Charge transfer plasmon (CTP) is a LSPR mode that strongly depends on a conductive junction between metallic nanounits. Benefitting from the charge transfer junction, CTP provides a facile way to generate widely tunable LSPR with highly localized/enhanced light magnetic field and photothermal effect. The limited availability of highly tunable CTP structures and their fabrication techniques hinders a further pursuit of their functions and applications. In response to this situation, the present work aims at developing a simple while highly efficient synthetic route to width-adjustable $\mathrm{Au} / \mathrm{Cu}$ heterojunctions capable of evoking tunable CTP behaviors. The strategy relies on a non-specific surface adsorption of low-cost, naturally occurred fish sperm DNA on a gold nanoseed to control heterogeneous copper nucleation. Such a process offers a chance to tailor the contact area between the gold and copper nano-domains in the bimetallic structure. Highly tunable CTP resonance from visible to near-infrared region is then realizable on the basis of this method. Experimental and calculated extinction spectra consistently reveal three key variables for the CTP structure, including the width of conductive junction and the sizes of gold and copper particles. These parameters are associated with DNA coverage, copper precursor concentration, and the synthetic conditions for gold nanoparticles, which allow for a CTP tuning from visible to near infrared wavelengths. By fully exploiting these highly controllable parameters, the maximally achievable CTP wavelength readily enters a near infrared II domain. The resulting CTP signals have a red-shift of up to $750 \mathrm{~nm}$ relative to the $530 \sim 570 \mathrm{~nm}$ LSPR peaks of individual gold and copper nanoparticles, corresponding to a very narrow $\mathrm{Au} / \mathrm{Cu}$ conductive contact of $11 \sim 13 \mathrm{~nm}$ in width. The role of nonspecific DNA adsorption in the above process proves unique (currently irreplaceable) compared to other molecular adsorbates. The easily tunable $\mathrm{Au} / \mathrm{Cu}$ heterointerface paves a way to integrated CTP and catalytic/sensing functions in future research.

Keywords charge transfer; interface; metal; dimerization; DNA
\end{abstract}

\section{1 引言}

局域表面等离激元共振(LSPR)是指在特定频率入
射光电场驱动下, 金属纳米材料表面发生的导带电子集 体相干振荡行为 ${ }^{[1]}$. 在具有纳米间隙的等离激元纳米粒 子二聚体中, 纵向 $\operatorname{LSPR}($ 成键偶极等离激元, 即 BDP)

*E-mail: zhxdeng@ustc.edu.cn

Received May 19, 2020; published June 29, 2020.

Supporting information for this article is available free of charge via the Internet at http://sioc-journal.cn.

Project supported by the National Key Research and Development Program of China (Nos. 2016YFA0201300, 2018YFA0702001) and the National Natural Science Foundation of China (Nos. 21425521, 21972130, 21521001).

项目受科技部重点研发计划(Nos. 2016YFA0201300, 2018YFA0702001)和国家自然科学基金(Nos. 21425521, 21972130, 21521001)资助. 
随粒子间隙变小而逐渐红移 ${ }^{[2-5]}$. BDP 模式可在纳米粒 子间隙处产生极强的光电场, 对光谱学和光催化等研究 十分有利, 但其频率一般局限于可见光波段. 当纳米粒 子间隙减小至量子隧穿域值时 $(<0.5 \mathrm{~nm})$ 将出现新的 LSPR 模式, 即电荷转移等离激元 $(\mathrm{CTP})^{[6]}$. 若粒子间进 一步形成欧姆接触, CTP 频率将随导电结宽度增加逐渐 蓝移 ${ }^{[6-9]}$. CTP 共振为近红外(NIR) I 和 II 区的 LSPR 调控 提供了机会, 并为测量导电结在光学频率下的电输运性 质提供了可能 ${ }^{[10]}$. 特别地, 窄的导电结可增强结点处电 流密度进而产生高度局域化的磁场增强和热效应(前者 对磁偶极光谱跃迁具有重要意义 ${ }^{[11]}$, 后者可加速化学反 应). 除了同质界面, 不同材料间形成的异质导电结还 可实现杂化的 CTP 共振, 并有利于调控界面处的光电 性质和催化活性 ${ }^{[12-22]}$. 然而, 获得可精确调控的异质电 荷转移界面, 尤其是窄导电结点, 在实验上十分困难.

光刻(或电子束刻蚀)常用于制造不同宽度的导电 结, 但其多晶结构会增加等离激元阻尼, 且难以构造三 维或异质结构. 此外, 光刻过程分辨率有限, 而电子束 刻蚀效率较低. 透射电子显微镜(TEM)的电子束或紫外 光照射可操控纳米粒子间发生可控融合 ${ }^{[23,24]}$, 但不适合 制备目的. 上述 “干态” 结构也不适合化学和生物溶液 体系的应用. 自组装是制备纳米二聚结构的常用方 法 ${ }^{[2,25-29]}$, 但组装结构难以发生粒子间电荷转移 ${ }^{[30-32]}$. 最近, Deng 等 ${ }^{[33-35]}$ 结合自组装与限域热(或激光)烧结, 制备出不同导电结宽度的二聚结构. 该方法不适合化学 活泼材料, 且高温下原子扩散加速会破坏异质界面. 相 比之下, 湿法过程更为灵活 ${ }^{[36]}$, 有望获得结晶良好的复 杂纳米结构, 并实现其低成本和规模化制备. 种子介导 化学沉积是实现 CTP 结构的另一类可行方法 ${ }^{[37-44]}$. Sun

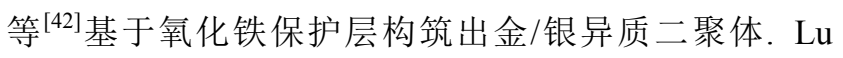
等 ${ }^{[43]}$ 利用不同 DNA 碱基的吸附能力差异, 基于纳米棒 再生长得到 CTP 哑铃结构. 然而, 获得窄的导电结点以 及宽广可调的异质 CTP 界面仍缺乏有效的方法. Nam 等 ${ }^{[44]}$ 以颈基 DNA “接枝” 的金纳米粒子(AuNP)为种子, 通过变化 $\mathrm{NaCl}$ 浓度调控 $\mathrm{Au} / \mathrm{Ag}$ “纳米雪人” 的导电结 点. 但是, 颈基 DNA 的高成本限制了制备量, 且未能拓 展至更多材料体系.

铜纳米粒子 $(\mathrm{CuNP})$ 是除了 $\mathrm{Au}$ 和 $\mathrm{Ag}$ 外, 在可见光区 表现出显著 LSPR 特性的第三种材料, 在微电子和催化 领域具有重要应用 ${ }^{[19,20,45-49]}$. 由于铜比金、银活泼, 在空 气中易被氧化, 其尺寸和形貌的化学合成控制相对困 难, 导致其等离激元调控应用较少. 本工作基于 DNA 分子非特异性吸附, 发展出一种简单、高效的 CTP 化学 调控方法, 通过改变 AuNP 种子表面 DNA 吸附量可定 量调控 $\mathrm{Au} / \mathrm{Cu}$ 纳米异质界面的结点宽度(图 1). DNA 吸 附层抑制了铜在 AuNP 表面的异相成核, 导致 $\mathrm{Au} / \mathrm{Cu}$ 接 触面减小并最终形成窄导电结点, 实现可见至 NIR I、II 区的 CTP 共振调控.

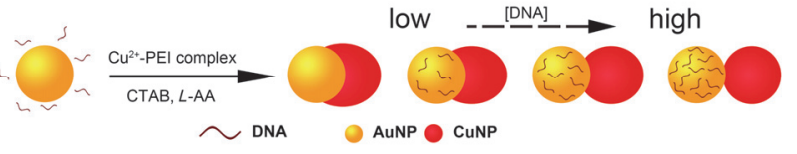

图 1 基于非特异性 DNA 吸附实现 $\mathrm{Au} / \mathrm{Cu}$ 纳米异质界面的高效调控. PEI, CTAB 和 $L$-AA 分别代表聚乙烯亚胺、十六烷基三甲基溴化铵和 $L$-抗坏血酸.

Figure 1 Highly tunable $\mathrm{Au} / \mathrm{Cu}$ heterointerface controlled by nonspecific DNA adsorption. PEI, CTAB, and $L$-AA stand for polyethylenimine, cetyltrimethylammonium bromide, and $L$-ascorbic acid, respectively.

\section{2 结果与讨论}

\section{$2.1 \mathrm{Au} / \mathrm{Cu}$ 纳米异质界面的构筑及 DNA 吸附调控}

由于我们的方法在原理上不依赖于特定 DNA 序列 或功能化基团(如末端巯基或硫代磷酸根), 因而可十分 方便地使用相对廉价的天然鱼精 DNA(FSDNA, 经机械 剪切处理以减小其分子量)作为吸附剂. 将 FSDNA 与柠 檬酸盐保护的 AuNP 预先作用一段时间, 便可作为种子 用于 $\mathrm{Au} / \mathrm{Cu}$ 异质结构制备. 该过程成功的关键之一是铜 在金表面发生异相成核，为保证这一点可加入聚乙烯亚 胺(PEI)与 $\mathrm{Cu}^{2+}$ 形成稳定的螯合物, 抑制 CuNP 自成 核 ${ }^{[50,51]}$. PEI 同时还可作为 CuNP 的表面配体, 减轻其氧 化和聚集现象 ${ }^{[50]}$. 实验发现，引入表面活性剂十六烷基 三甲基溴化铵(CTAB)有利于生成尺寸相对均匀的准球 形 CuNP. 粉末 X 射线衍射(XRD)证明所得产物包含金、 铜两种结晶相(图 S1).



图 2 FSDNA 调控 $\mathrm{Au} / \mathrm{Cu}$ 异质界面导电结点宽度所得产物的透射电 镜图像. 1 和 2 分别对应 25 和 $40 \mathrm{~nm}$ AuNP 种子. $\mathrm{a} 1 \sim \mathrm{d} 1$ : FSDNA 浓度 依次为 $0 、 0.02 、 0.04$ 和 $0.10 \mathrm{~g} \cdot \mathrm{L}^{-1} ; \mathrm{a} 2 \sim \mathrm{d} 2:$ FSDNA 浓度依次为 0 、 $0.01 、 0.03$ 和 $0.10 \mathrm{~g} \cdot \mathrm{L}^{-1}$. 内插图为所得结构的高倍电镜图像.

Figure 2 TEM images of $\mathrm{Au} / \mathrm{Cu}$ heterodimers with tunable interparticle junctions controlled by FSDNA. Rows $1 \sim 2$ correspond to increased diameters ( 25 and $40 \mathrm{~nm}$, respectively) of Au nanoseeds. Columns a-d represent increased FSDNA concentrations: $0,0.02,0.04$, and $0.10 \mathrm{~g} \cdot \mathrm{L}^{-1}$ for panels a1 $\sim \mathrm{d} 1$; and $0,0.01,0.03$, and $0.10 \mathrm{~g} \cdot \mathrm{L}^{-1}$ for panels $\mathrm{a} 2 \sim \mathrm{d} 2$. Insets are enlarged views of representative structures.

透射电子显微(TEM)成像清楚揭示了不同 DNA 吸 附量和 AuNP 尺寸对 $\mathrm{Au} / \mathrm{Cu}$ 异质结构的调控作用(图 2). 
图中可见, $\mathrm{Au} / \mathrm{Cu}$ 异质二聚体纯度很高, 且未沉积 $\mathrm{Cu}$ 的 AuNP 种子和均相成核生成的 CuNP 副产物十分少见. 更为重要的是, 随 FSDNA 浓度升高, AuNP 和 CuNP 之 间的异质结点规律性变窄. 由于 CuNP 的电子衬度低于 AuNP, 从 TEM 图像可清晰分辨二者间的异质界面. 基 于电镜图像, 我们测量了 $\mathrm{Au} / \mathrm{Cu}$ 二聚体的结点宽度和对 应 CuNP 尺寸(图 S2, S3). 结果表明: 增加 FSDNA 吸附 量可将二聚体结点宽度减小至 $11 \sim 13 \mathrm{~nm}$ 左右, 而 CuNP 尺寸变化不大; 随着 AuNP 种子增大, 异质界面 宽度仅略有增加. 研究还发现, 高浓度 FSDNA 可帮助 改善产物均一性, 这可能与 DNA 在 CuNP 表面吸附有 关, 该现象对制备窄结点宽度的异质结构十分有利. 通 过改变铜盐浓度, 可进一步调控二聚体中 CuNP 的尺寸, 图 3 为较高 FSDNA 浓度下所得二聚体的电镜图像. 可 以看出, 二聚体中 CuNP 尺寸与铜盐浓度呈现正相关. 固定 DNA 浓度, CuNP 随 $\mathrm{Cu}^{2+}$ 浓度增加而变大, 但异质 结点宽度几乎不变(图 S4, S5), 这种 DNA 和 $\mathrm{Cu}^{2+}$ 调控的 正交性为 $\mathrm{Au} / \mathrm{Cu}$ 异质结构的定制合成提供了便利.



图 3 不同 $\mathrm{Cu}$ 沉积量对应 $\mathrm{Au} / \mathrm{Cu}$ 异质二聚体的透射电镜图像. 1 和 2 分别对应 25 和 $40 \mathrm{~nm} \mathrm{AuNP}$ 种子. $\mathrm{a} \sim \mathrm{c}$ 对应依次增加的 $\mathrm{Cu}^{2+}$ 浓度. FSDNA 浓度固定为 $0.10 \mathrm{~g} \cdot \mathrm{L}^{-1}$. 内插图为相应结构的高倍电镜图像.

Figure 3 TEM images of $\mathrm{Au} / \mathrm{Cu}$ heterodimers with tunable $\mathrm{Cu}$ domain sizes dependent on $\mathrm{Cu}^{2+}$ concentration. Rows $1 \sim 2$ correspond to 25 and $40 \mathrm{~nm} \mathrm{Au}$ nanoseeds, respectively. Columns $\mathrm{a} \sim \mathrm{c}$ represent increased $\mathrm{Cu}^{2+}$ concentrations. FSDNA concentration is $0.10 \mathrm{~g} \cdot \mathrm{L}^{-1}$ in all cases. Insets are enlarged views of representative structures.

\section{$2.2 \mathrm{Au} / \mathrm{Cu}$ 异质结构的电荷转移等离激元共振性质}

根据等离激元理论, 纳米二聚结构的电荷转移 LSPR 波长对粒子间结点的导电性非常敏感 ${ }^{[2,4,10]}$. 为进 一步探究金、铜纳米粒子间导电通道的性质, 我们测量 了具有不同结点宽度的 $\mathrm{Au} / \mathrm{Cu}$ 异质二聚体的可见-近红 外消光光谱(图 $4 \mathrm{a} \sim 4 \mathrm{~d}$ ). 结果可见, 对于较宽结点的异 质二聚体(对应最低非零 FSDNA 浓度), 在 $615 \mathrm{~nm}$ 处出 现一个 LSPR 肩峰(图 4a). 随着结点宽度减小, 该峰位
置逐渐红移至 $900 \mathrm{~nm}$ 处. 该现象说明金、铜之间形成了 良好的欧姆接触，其导电性随结点宽度变窄而降低，对 应电荷转移等离激元共振频率不断红移, 这与已有理论 很好吻合 ${ }^{[4]}$. 对于更宽的结点(对应未经 FSDNA 吸附的 AuNP 种子), CTP 与横向共振频率接近而难以分辨, 消 光光谱显示 AuNP 和 CuNP 的重叠 LSPR 峰(分别在 530 和 $570 \mathrm{~nm}$ 左右). 对比图 $4 \mathrm{a}$ 和 $4 \mathrm{c}$ 可知, 增加 AuNP 尺寸 可以得到 CTP 更为红移的消光光谱, 其极限波长由 900 $\mathrm{nm}$ 增加到 $1150 \mathrm{~nm}$. 这是因为 CTP 频率与结点宽度 $\left(L_{\mathrm{CJ}}\right)$ 和纳米粒子直径 $(d)$ 的比值 $\left(L_{\mathrm{CJ}} / d\right)$ 直接相关. 类似地, 随 着 CuNP 增大, CTP 波长亦更为红移 (图 4b, 4d). 对于 40 $\mathrm{nm}$ 的 AuNP 种子, 通过增大 CuNP 可使其 CTP 红移至 $>1300 \mathrm{~nm}$ 处. 为进一步理解光谱性质与等离激元结构 间的关系，我们使用边界元法对不同 $\mathrm{Au} / \mathrm{Cu}$ 异质结构 (图 4a, 4b)的消光光谱(吸收和散射之和)进行了计算 ${ }^{[52]}$, 所得理论光谱与实验结果非常一致(图 4e, 4f). 为保证 计算结果的说服力, 所建模型的几何参数与电镜测量值 尽量一致. 计算结果很好证明了结点宽度(图 4e) 和 CuNP 尺寸(图 4f) 与电荷转移共振频率间的直接关联,
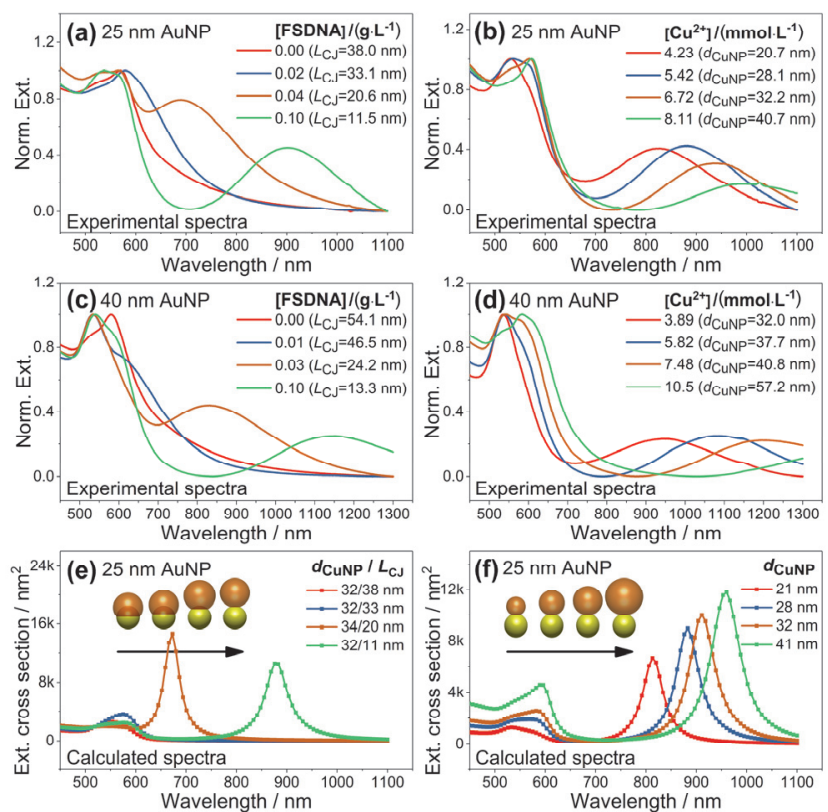

图 4 不同 $\mathrm{FSDNA}$ 和 $\mathrm{Cu}^{2+}$ 浓度调控所得 $\mathrm{Au} / \mathrm{Cu}$ 异质结构的消光光谱. (a, b) 使用 $25 \mathrm{~nm} \mathrm{AuNP}$ 种子所得结构. (c, d) $40 \mathrm{~nm}$ AuNP 种子所得结 构. (e, f) 边界元法计算所得 $25 \mathrm{~nm} \mathrm{AuNP}$ 种子对应结构的纵向等离激 元理论消光光谱 (与 $\mathrm{a}, \mathrm{b}$ 对应). DNA 和 $\mathrm{Cu}^{2+}$ 浓度以及相应结构的几何 参数 $\left(d_{\mathrm{CuNP}}\right.$ 和 $L_{\mathrm{CJ}}$ 分别为 $\mathrm{Cu}$ 粒子直径和 $\mathrm{Au} / \mathrm{Cu}$ 导电接触界面的弧长) 均见图中标注. (f)中所有模拟均设定结点宽度为 $10 \mathrm{~nm}$.

Figure 4 Extinction spectra of $\mathrm{Au} / \mathrm{Cu}$ heterodimers synthesized in the presence of different concentrations of FSDNA (a, c) and $\mathrm{Cu}^{2+}(\mathrm{b}, \mathrm{d})$. AuNP seeds with diameters of $25 \mathrm{~nm}(\mathrm{a}, \mathrm{b})$ and $40 \mathrm{~nm}(\mathrm{c}, \mathrm{d})$ are employed. (e, f) Calculated extinction cross sections for structures in $(a, b)$ by a boundary element method. Concentrations of FSDNA and $\mathrm{Cu}^{2+}$ as well as geometrical parameters $\left(d_{\mathrm{CuNP}}\right.$ and $L_{\mathrm{CJ}}$ represent the diameter of CuNPs and the arc length of $\mathrm{Au} / \mathrm{Cu}$ conductive contacts) of the heterodimers are indicated on corresponding panels. Junction widths are $10 \mathrm{~nm}$ for structures in (f). 
且前者更为关键. 理论结果同时显示, 由于异质二聚体 粒径较小, 其消光光谱主要来自吸收截面的贡献(图 S6).

以上实验表明，基于三种灵活的变量控制(AuNP 尺 寸、DNA 浓度和 $\mathrm{Cu}^{2+}$ 浓度), 可以实现 $\mathrm{Au} / \mathrm{Cu}$ 纳米异质 界面的按需定制, 获得可见至近红外 I、II 区的电荷转移 等离激元共振. 该结果为对入射光波长有严格要求的光 热治疗、光催化等研究提供了机会. 值得一提的是, 上 述方法合成的 $\mathrm{Au} / \mathrm{Cu}$ 异质结构具有很好的胶体稳定性, 可耐受 $-20{ }^{\circ} \mathrm{C}$ 冷冻保存以减缓其化学氧化. 解冻后的 样品可保持原始的光学性质和形貌(图 S7, S8), 为进一 步探索其相关功能与应用提供了便利.

\subsection{DNA 吸附调控作用的独特性}

基于以上实验结果, 可以推断 DNA 在 AuNP 表面 的吸附作用是调控 $\mathrm{Au} / \mathrm{Cu}$ 异质界面最为关键的因素. 为 验证这一机制, 我们在实验中有意省略 FSDNA 与柠檬 酸盐保护 AuNP 的预吸附等待步骤. 图 S9a, 9b 可见, 与 预吸附 $5 \mathrm{~min}$ 相比, 免除预吸附等待过程的 $\mathrm{Au} / \mathrm{Cu}$ 二聚 结构具有明显更宽的异质界面. 此外, 考虑到有机膦配 体(双(对-磺酸苯基)苯基膦, BSPP)与金表面作用强于 DNA 吸附, 我们还使用 BSPP 修饰的 AuNP 作为种子合 成 $\mathrm{Au} / \mathrm{Cu}$ 异质结构, 以进一步证明 DNA 吸附是结点宽 度调控的关键. 图 S9c, 9d 显示, 即使将预吸附时间延长 至 $>10 \mathrm{~h}$, 所得产物的结点宽度仍无明显变窄. 以上实 验揭示 AuNP 表面预先形成的 DNA 吸附层是调控导电 结点宽度的关键因素, 其可有效抑制铜在金表面异相成 核, 进而减小 $\mathrm{Au} / \mathrm{Cu}$ 接触界面.

利用非特异性 DNA 吸附实现 $\mathrm{Au} / \mathrm{Cu}$ 异质界面的有 效调控, 其优势在于无需使用序列确定的人工合成 DNA, 在保证调控简便性的同时, 大大降低了制备成 本. DNA 在 AuNP 表面另一种常见的吸附模式是通过 金一颈基相互作用. 非特异性吸附的 DNA 一般 “平躺” 于纳米粒子表面，而放基修饰的 DNA 则倾向于 “站立” 状态 ${ }^{[53]}$. 为对比此两种模式对 $\mathrm{Au} / \mathrm{Cu}$ 异质结构的影响, 我们分别使用具有颈基末端和不含颈基的人工合成 DNA 对 AuNP 进行修饰, 研究其对 $\mathrm{Au} / \mathrm{Cu}$ 结点的调控 作用(图 S10 S12). 结果显示: 非放基 DNA 可类似调 节导电结点宽度; 而放基 DNA 仅在高密度修饰时才能 得到较窄的导电结点, 增加 DNA 链长并无进一步改善. 值得指出的是, 由于非特异性吸附作用较弱, 且 DNA 分子平躺于表面, 其吸附密度和吸附层厚度均远低于䘪 基 DNA.

除 DNA 外, 我们还选择了其它一些吸附剂分子, 包括聚乙烯吡咯烷酮(PVP)、聚丙烯酸(PAA)、鱼精蛋白 以及 4-䘪基苯乙酸(MPAA), 结果显示这些大分子和小 分子均无可见的调控效果(图 S13, S14). 尽管非特异性 DNA 吸附对 $\mathrm{Au} / \mathrm{Cu}$ 异质界面的调控作用十分显著, 其 对 $\mathrm{Au} / \mathrm{Ag}$ 异质界面的调控却相对有限(图 S15, S16), 难
以形成窄导电结点, 这可能与 $\mathrm{Au}$ 、 $\mathrm{Ag}$ 之间 “亲和性” 更强有关. 由此可见，非特异性 DNA 吸附对 $\mathrm{Au} / \mathrm{Cu}$ 异 质界面具有十分高效且独特的调控作用, 通过与其它调 控方式结合有望实现更为复杂的等离激元结构.

\section{3 结论}

本工作发展了一种简单且高效的 $\mathrm{Au} / \mathrm{Cu}$ 纳米异质 界面电荷转移共振调控方法. 利用 DNA 分子在 $\mathrm{Au}$ 纳米 粒子表面的非特异性吸附改变金属 $\mathrm{Cu}$ 在 $\mathrm{Au}$ 表面的异 相成核特性, 进而实现 $\mathrm{Au} / \mathrm{Cu}$ 异质导电结点的宽度控 制. 在此基础上获得可见至近红外 I、II 区电荷转移共振 的宽广调控，且实验光谱与理论预测相吻合. 该方法不 依赖于特定 DNA 序列或化学修饰, 可使用相对廉价、 易得的天然 DNA 分子, 为降低成本和扩大制备规模奠 定了基础. 研究还揭示了 DNA 吸附调控作用的独特性 (相对于其它分子), 并有望推广至更多同质和异质体系, 为电荷转移纳米结构的直接合成以及纳米成核机理研 究提供了重要机会. 除 DNA 等大分子外，小分子吸附 剂也值得研究 ${ }^{[54]}$. 本工作所得结构与纳米棒相比具有 显著区别: (i) CTP 共振受粒子间界面调控, 为研究特定 导电结点在光频率下的电输运提供了可能; (ii) CTP 可 在结点处产生局域磁场增强, 为磁偶极光谱调控提供了 机会; (iii) CTP 对结点材料响应灵敏且不同结构体积相 似，可为传感和纳米生物效应研究提供更合适的材料. 考虑到电荷转移等离激元在光学传感、光捕获、光电器 件和局域磁/热增强等方面的特殊意义，以及金属异质 界面在催化方面的重要性，本工作发展的方法和纳米结 构为上述功能与应用的探索奠定了基础.

\section{References}

[1] Hutter, E.; Fendler, J. H. Adv. Mater. 2004, 16, 1685.

[2] Halas, N. J.; Lal, S.; Chang, W. S.; Link, S.; Nordlander, P. Chem. Rev. 2011, 111, 3913.

[3] Nordlander, P.; Oubre, C.; Prodan, E.; Li, K.; Stockman, M. I. Nano Lett. 2004, 4, 899.

[4] Romero, I.; Aizpurua, J.; Bryant, G. W.; de Abajo, F. J. G. Opt. Express 2006, 14, 9988.

[5] Rechberger, W.; Hohenau, A.; Leitner, A.; Krenn, J. R.; Lamprecht, B.; Aussenegg, F. R. Opt. Commun. 2003, 220, 137.

[6] Savage, K. J.; Hawkeye, M. M.; Esteban, R.; Borisov, A. G.; Aizpurua, J.; Baumberg, J. J. Nature 2012, 491, 574.

[7] Esteban, R.; Borisov, A. G.; Nordlander, P.; Aizpurua, J. Nat. Commun. 2012, 3, 825 .

[8] Wen, F. F.; Zhang, Y.; Gottheim, S.; King, N. S.; Zhang, Y.; Nordlander, P.; Halas, N. J. ACS Nano 2015, 9, 6428.

[9] Atay, T.; Song, J. H.; Nurmikko, A. V. Nano Lett. 2004, 4, 1627.

[10] Pérez-González, O.; Zabala, N.; Borisov, A. G.; Halas, N. J.; Nordlander, P.; Aizpurua, J. Nano Lett. 2010, 10, 3090.

[11] Grosjean, T.; Mivelle, M.; Baida, F. I.; Burr, G. W.; Fischer, U. C. Nano Lett. 2011, 11, 1009.

[12] Lim, B. K.; Kobayashi, H.; Yu, T.; Wang, J. G.; Kim, M. J.; Li, Z. Y.; Rycenga, M.; Xia, Y. N. J. Am. Chem. Soc. 2010, 132, 2506.

[13] Tao, Z. X.; Wu, Z. S.; Yuan, X. L.; Wu, Y. S.; Wang, H. L. ACS Catal. 2019, 9, 10894.

[14] Morales-Guio, C. G.; Cave, E. R.; Nitopi, S. A.; Feaster, J. T.; Wang, L.; Kuhl, K. P.; Jackson, A.; Johnson, N. C.; Abram, D. N.; Hatsukade, T.; Hahn, C.; Jaramillo, T. F. Nat. Catal. 2018, 1, 764.

[15] Zhu, X. Z.; Yip, H. K.; Zhuo, X. L.; Jiang, R. B.; Chen, J. L.; Zhu, 
X.-M.; Yang, Z.; Wang, J. F. J. Am. Chem. Soc. 2017, 139, 13837.

[16] Kortlever, R.; Peters, I.; Balemans, C.; Kas, R.; Kwon, Y.; Mul, G.; Koper, M. T. M. Chem. Commun. 2016, 52, 10229.

[17] Cai, Z.; Kuang, Y.; Luo, L.; Wang, L. R.; Sun, X. M. Acta Chim. Sinica 2013, 71, 1265 (in Chinese). (蔡到, 广允, 罗亮, 王利人, 孙晓明，化学学报, 2013, 71, 1265.)

[18] Liu, B. L.; Zhang, H. C.; Ding, Y. Chin. Chem. Lett. 2018, 29, 1725.

[19] Huang, J.; Mensi, M.; Oveisi, E.; Mantella, V.; Buonsanti, R. J. Am. Chem. Soc. 2019, 141, 2490.

[20] Huang, X.; Li, Y.; Zhou, H.; Zhong, X.; Duan, X.; Huang, Y. Chem. Eur. J. 2012, 18, 9505.

[21] Wu, K. H.; Zhou, Y. W.; Ma, X. Y.; Ding, C.; Cai, W. B. Acta Chim. Sinica 2018, 76, 292 (in Chinese). (吴匡衡, 周亚威, 马宪印, 丁 辰, 蔡文斌, 化学学报, 2018, 76, 292.)

[22] Xu, S. Y.; Liu, Z. H.; Zhang, H.; Yu, J. R. Acta Chim. Sinica 2019, 77,427 (in Chinese). (徐姝雅, 刘治宏, 张淮, 于金再, 化学学报, 2019, 77, 427.)

[23] Jung, H.; Cha, H.; Lee, D.; Yoon, S. ACS Nano 2015, 9, 12292.

[24] Scholl, J. A.; Garcia-Etxarri, A.; Koh, A. L.; Dionne, J. A. Nano Lett. 2013, 13, 564 .

[25] Jones, M. R.; Osberg, K. D.; Macfarlane, R. J.; Langille, M. R.; Mirkin, C. A. Chem. Rev. 2011, 111, 3736.

[26] Lan, X.; Chen, Z.; Liu, B. J.; Ren, B.; Henzie, J.; Wang, Q. B. Small 2013, 9, 2308.

[27] Zhong, Z. Y.; Patskovskyy, S.; Bouvrette, P.; Luong, J. H. T.; Gedanken, A. J. Phys. Chem. B 2004, 108, 4046.

[28] Maye, M. M.; Nykypanchuk, D.; Cuisinier, M.; van der Lelie, D.; Gang, O. Nat. Mater. 2009, 8, 388.

[29] Yu, H.; Man, T. T.; Ji, W.; Shi, L. L.; Wu, C. W.; Pei, H.; Zhang, C. Chin. Chem. Lett. 2019, 30, 175.

[30] Kim, J.-Y.; Kotov, N. A. Chem. Mater. 2014, 26, 134.

[31] Li, Y. L.; Deng, Z. X. Acc. Chem. Res. 2019, 52, 3442.

[32] Song, L.; Deng, Z. X. ChemNanoMat 2017, 3, 698.

[33] Fang, L. L.; Wang, Y. L.; Liu, M.; Gong, M.; Xu, A.; Deng, Z. X. Angew. Chem. Int. Ed. 2016, 55, 14296.

[34] Fang, L. L.; Liu, D. L.; Wang, Y. L.; Li, Y. J.; Song, L.; Gong, M.; Li, Y.; Deng, Z. X. Nano Lett. 2018, 18, 7014.

[35] Liu, M.; Fang, L. L.; Li, Y. L.; Gong, M.; Xu, A.; Deng, Z. X. Chem. Sci. 2016, 7, 5435 .
[36] Wang, Y. L.; Fang, L. L.; Gong, M.; Deng, Z. X. Chem. Sci. 2019, $10,5929$.

[37] Sun, Y. Natl. Sci. Rev. 2015, 2, 329.

[38] Gu, H. W.; Yang, Z. M.; Gao, J. H.; Chang, C. K.; Xu, B. J. Am. Chem. Soc. 2005, 127, 34.

[39] Zhu, C.; Zeng, J.; Tao, J.; Johnson, M. C.; Schmidt-Krey, I.; Blubaugh, L.; Zhu, Y. M.; Gu, Z. Z.; Xia, Y. N. J. Am. Chem. Soc. 2012 134, 15822.

[40] Yu, H.; Chen, M.; Rice, P. M.; Wang, S. X.; White, R. L.; Sun, S. H. Nano Lett. 2005, 5, 379.

[41] Feng, Y. H.; He, J. T.; Wang, H.; Tay, Y. Y.; Sun, H.; Zhu, L. F.; Chen, H. Y. J. Am. Chem. Soc. 2012, 134, 2004.

[42] Sun, Y. G.; Foley, J. J.; Peng, S.; Li, Z.; Gray, S. K. Nano Lett. 2013, $13,3958$.

[43] Song, T. J.; Tang, L. H.; Tan, L. H.; Wang, X. J.; Satyavolu, N. S. R.; Xing, H.; Wang, Z. D.; Li, J. H.; Liang, H. J.; Lu, Y. Angew. Chem. Int. Ed. 2015, 54, 8114.

[44] Lee, J. H.; You, M. H.; Kim, G. H.; Nam, J. M. Nano Lett. 2014, 14, 6217.

[45] Gawande, M. B.; Goswami, A.; Felpin, F. X.; Asefa, T.; Huang, X. X.; Silva, R.; Zou, X. X.; Zboril, R.; Varma, R. S. Chem. Rev. 2016, $116,3722$.

[46] Chen, S. T.; Jenkins, S. V.; Tao, J.; Zhu, Y. M.; Chen, J. Y. J. Phys. Chem. C 2013, 117, 8924.

[47] Osowiecki, W. T.; Ye, X. C.; Satish, P.; Bustillo, K. C.; Clark, E. L.; Alivisatos, A. P. J. Am. Chem. Soc. 2018, 140, 8569.

[48] Wu, S. H.; Chen, D. H. J. Colloid Interface Sci. 2004, 273, 165.

[49] Lin, M. H.; Kim, G. H.; Kim, J. H.; Oh, J. W.; Nam, J. M. J. Am. Chem. Soc. 2017, 139, 10180.

[50] Kim, J. H.; Park, J. E.; Lin, M.; Kim, S.; Kim, G. H.; Park, S.; Ko, G.; Nam, J. M. Adv. Mater. 2017, 29, 1702945.

[51] Kislenko, V. N.; Oliynyk, L. P. J. Polym. Sci., Part A: Polym. Chem. 2002, 40, 914.

[52] Hohenester, U.; Trügler, A. Comput. Phys. Commun. 2012, 183, 370.

[53] Wolf, L. K.; Gao, Y.; Georgiadis, R. M. Langmuir 2004, 20, 3357.

[54] Fang, Y. J. Chem. Phys. 1998, 108, 4315.

(Cheng, B.; Fan, Y.) 